\title{
Computer Aided Diagnosis of Dental CT images for Bone Quality Assessment
}

\author{
Dr. T. Kalpalatha Reddy, T. Kaliraja, B. Ramesh, and G. Gurumurthy
}

\begin{abstract}
The objective of this paper is to design and implement classifier framework to assist the surgeon for preoperative assessment of bone quality from Dental Computed Tomography images. This article focuses on comparing the discriminating power of several multiresolution texture analysis methods to evaluate the quality of the bone based on the texture variations of the images obtained from the implant site using wavelet, curvelet and contourlet.The approach consists of three steps: automatic extraction of the most discriminative texture features of regions of interest, creation of a classifier that automatically grades the bone depends on the quality and correlating significant texture parameters with Insertion Torque Values. Since this is medical domain, the validation against the human experts is carried out. The results indicate that the combination of the statistical and multiscale representation of the bone image gives adequate information to classify the different bone groups compared to gray level features at single scale.
\end{abstract}

Index Terms-Multiresolution analysis, texture classification, wavelets, ridgelets, curvelets, contourlets, dental computed tomography.

\section{INTRODUCTION}

Implant dentistry is the treatment of choice to replace missing teeth in both partially and completely edentulous patients [1],[2]. Bone quality was considered as a critical parameter associated with implant success rate.Pre-operative evaluation of bone density is essential to assist the clinician with the treatment planning of implant therapy.The first problem faced by the dental surgeons is all the present pre -implant radiographic methods do not provide information on the quality of the bone at proposed implant site. The surgeons will know about the bone quality present in the jaw only at the time of explorative drilling in fixture site preparation.

implant immediately after extraction of the tooth and to place the prosthetic restoration on the day of implant surgery known as 'immediate loading'.

At present, clinically available dental CT does not provide sufficient resolution to resolve trabecular structures. Modern dental CT scanners for measurements of the axial skeleton offer isotropic spatial resolution of approximately $0.5 \mathrm{~mm}$. However, given the typical dimensions of trabeculae $(100-400 \mu \mathrm{m})$ and trabecular spaces $(200-2000 \mu \mathrm{m})$, this resolution is not sufficient for a direct determination of

Mamuscript received September 16, 2011; revised September 30, 2011.

Dr. T. Kalpalatha Reddy, T. Kaliraja, B. Ramesh and G Gurumurthy are with the Department of Electronics and Communications Engineering,SKR Engineering College affiliated to Anna University, Chennai, India (Email-drkalpalatha.thokala@gmail.com). trabecular architecture and due to substantial partial volume effects, the extraction of the quantitative structural information is difficult and the results vary substantially according to the threshold and image processing techniques. Therefore, instead of measuring structural parameters directly there is a trend to use textural or statistical descriptors to characterize the trabecular architecture without requiring stringent segmentation of the individual trabeculae.Thus the methods described in this paper will allow estimates of structural parameters from CT images, providing for the first time, the possibility of clinical use of such estimates[3].

\section{Bone Quality}

Bone quality is dependent on bone density and bone quantity.Lekholm and Zarb (1985) classified bone quality into four types (Fig. 1).

Quality1 (D1)-comprises homogeneous and compact bone.

Quality2 (D2)-a thick layer of compact bone surrounding a core of dense trabecular bone.

Quality3 (D3)-a thin layer of cortical bone surrounding dense trabecular bone of favorable strength.

Quality4 (D4)-a thin layer of cortical bone surrounding a core of low-density trabecular bone.

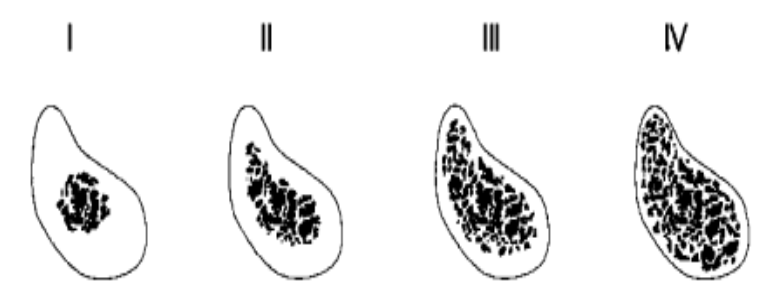

Fig. 1. Classification of Bone Quality according to Lekholm and Zarb (1985)

Co-occurrence matrices are often used in texture analysis since they are able to capture the spatial dependence of gray level values within an image. Run length matrices are able to capture the coarseness of texture in specified directions as defined by gray level runs. The research focuses on multi-resolution analysis of multi wavelet taken in to account the impact of spatial distribution of pixels and the threshold transacting through the transformation coefficients at different resolution levels. It offers a comprehensive analysis of four forms of multi-resolution analysis, as well as a comparison with three standard texture classification algorithms: Histogram, co-occurrence and run length.

Multiresolution analysis has been successfully used in image processing with the recent emergence of applications to texture classification[5],[6]. Several studies have investigated the discriminating power of wavelet-based features in various applications including: image compession, denoising and classification of textures. Inspired by the success of wavelets, a number of new multiresolution analysis tools like contourlet, 
ridgelet and curvelets etc have been developed to resolve directional features. The traditional multiresolution methods keep an eye on the spectral information of the texture image at different scales and use the statistics of the spectral information as the texture descriptor but they ignore the texture structural information. To make use of the texture primitives, histogram and GLCM are combined with the traditional multiresolution method so that a better classification of bone quality becomes possible[3]. The role of multiresolution based statistical methods in the improvement of the classification is studied in this work. The overall scheme of the work is as shown in Fig. 2.

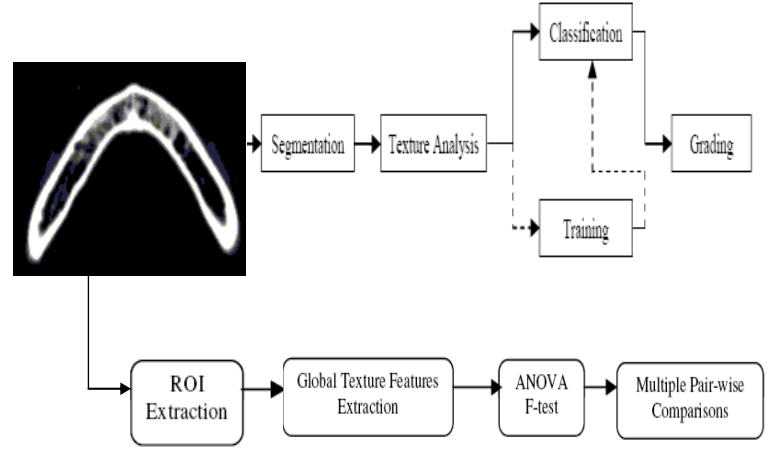

Fig. 2. The Scheme of Texture-based classification system

The appropriate multi-resolution transform was applied and a set of texture descriptors was extracted from the transformed image. These features characterized the textural properties of the images and were used to train the classifier to recognize each texture class. The classification step was implemented through a decision tree classifier, Support Vector Machine and Adaboost based on the cross-validation approach. The testing test was then fed through the classification system, resulting in a misclassification matrix from which performance measures were calculated[9],[10].

The paper is structured as follows: Section 2 describes in more detail the multi-resolution analysis and summarizes the discrete forms of wavelet, ridgelet, curvelet and contourlet transforms. Section 3 discusses the overall methodology, as well as the classifier and performance measures. Section 4 presents a comparison of the results.

\section{Multi-Resolution AnAlysis}

Multi-resolution analysis of an image is important in characterization of texture by capturing the underlying intensity variations.Currently the wavelet transform and the Gabor transform are the most popular multiresolution methods. Compared to the wavelet transform, the Gabor transform needs to select the filter parameters according to different texture. Wavelets provide a very sparse and efficient representation for piecewise smooth signals having finite singularities. But it cannot efficiently represent discontinuities along edges or curves in images or objects except in horizontal and vertical directions. Wavelets rely on isotropic scaling and have limited abilities to resolve directional features. The good performance of wavelets in one- dimensional domain is lost when they are applied to images using two-dimensional (2D) separable basis since they are not able to efficiently code one-dimensional singularities[11],[12].

To overcome the restriction, the Radon transform is introduced, and the combination of Radon transform and the wavelet has made a great progress in texture analysis. The Radon transform is used to detect the principal direction of the texture. Then the texture is rotated to place its principal direction at 0 degree. A wavelet transform is applied to the rotated image to extract texture features. Magli et al (1998) applied radon transform and 1-D continuous wavelet transform to detect linear patterns in the aerial images.

Candes and Donoho (1999) developed a system of representations named Ridgelet, which deal effectively with line singularities in 2-D. The idea suggested, is to map a line singularity into a point singularity using the Radon transform. Then, the translation invariant wavelet transform is applied to calculate the frequency components and extract the corresponding features. This technique preserves the orthogonality of the basis functions of the wavelet transform which makes the extracted features uncorrelated and allows classification using fewer number of features. One limitation to this approach is that ridgelets are more effective in detecting linear radial structures, which are not dominant in medical images[15],[16].

Curvelet transform is a recent entry in this category of multiscale multidirectional transform with better directional capabilities compared to wavelet transform. Conceptually, this mathematical tool is a multiscale pyramid with many directions and position at each length scale and needle shaped elements at fine scales [11],[12]. Like ridgelets, curvelets can occur at any scale, location and orientation [20],[21],[22]. But curvelets have variable length in addition to variable width (variable anisotropy), whereas ridgelets have only variable width and global length.

Curvelet is a block based transform, either the approximated images have blocking effects or due to the use of overlapping windows it leads to increase in redundancy. Secondly the use of the ridgelet transform, which is defined on a polar coordinate, makes the implementation of the curvelet transform for discrete images on a rectangular coordinate to be very challenging. Inspired by curvelets, Do and Vetterli (2002) developed the contourlet representation based on efficient two-dimensional non-separable filter banks that can deal effectively with images having smooth contours. Contourlets possess not only the main features of wavelets, namely multi resolution and time-frequency localization, but they also show a high degree of directionality and anisotropy. This application requires the use of discrete versions of the transforms which are still the subject of current research[13],[14].

\section{METHODOLOGY}

The texture classification algorithm consists of three main steps: segmentation of regions of interest, extraction of the most discriminative texture features, creation of a classifier that automatically identifies the various bone groups and correlating significant texture parameters with insertion torque.The following section describes the data set and 
section 3.2 describes the texture extraction methods. Section 3.3 describes the classifiers and performance measures used in the classification.

\section{A. The data set}

Dental CT is ideally suited for applying dose reduced investigation protocols. The possibility of reducing the dose is to limit the scan range by excluding the occlusal region. Single, partial, or total edentulous patients were included. Cross sectional images were acquired from each patient for assessment of the intended surgical areas including any pathological conditions and to evaluate the width and height of the crestal bone. The images were acquired with multi slice spiral CT scans $(120 \mathrm{kV}$ and $300 \mathrm{mAs}$, with a slice thickness of $0.625 \mathrm{~mm}$, pitch $0.4 \mathrm{~mm}$, scan time $750 \mathrm{msi}$ GE medical systems). The 3D DICOM image data consists of 190 consecutive 2D slices, each slice being $512 \times 512$ pixels in size and having 16 bit gray level resolution.

Wavelets,curvelets and contourlets are extremely sensitive to contrast in gray-level intensity.Therfore in order to use multiresolution based texture features, it was necessary to eliminate all background pixels to avoid mistaking the edge between artificial background and the organ as a texture feature. Each slice is therefore further cropped to the respective sizes because of size requirements of $2^{\text {n }}$ for wavelets and a prime size for ridgelets. Sixty five patients referred for single or multiple tooth implant treatment at the incisor, canine or premolar regions of the maxilla or the mandible are enrolled in this study (Table 3.2 for implant sites). All surgery was undertaken by one surgeon who also evaluated the bone quality according to the classification described by Lekholm and Zarb (1985). Fig.6.1, Fig.6.2 and Fig.6.3 shows an example of dental CT scan and a processed and cropped slice of the bone.
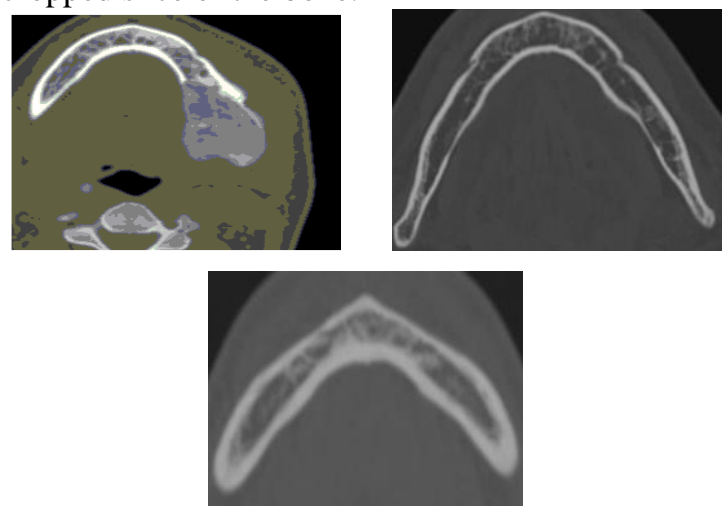

Fig. 3.Axial View of Maxilla and Mandible CT images

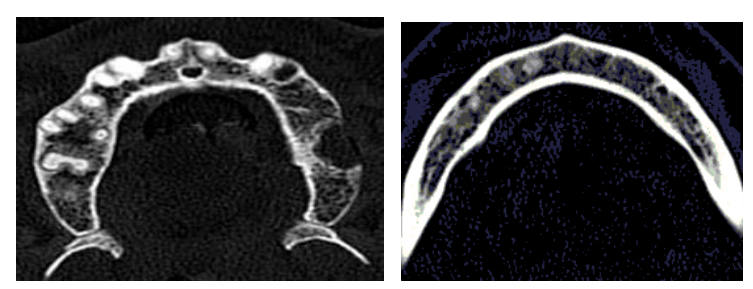

Fig. 4.Enhanced Bone Images (Background Removed)

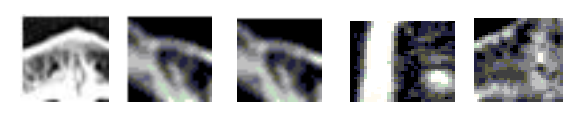

Fig. 5. Recipient Implant Sites (ROI)

\section{B. Feature extraction}

Once the medical images were pre-processed as described in section 3.1, the following multiresolution transforms were applied and textural feature vectors were then extracted: Gabor wavelet, Coiflet wavelet, ridgelet, curvelet and contourlet .After the transforms were applied, first and second order statistics were extracted for use in classification.

1) Discrete wavelet transform

For each image pixel, the Gabor transform leads to two kinds of Gabor features: Gabor magnitude features and Gabor phase features. In this frame work, the energy of the output of a bank of Gabor filters has been used as features for identifying the bone texture. In this work Gabor filters are oriented in 8 directions spaced equidistantly between 0 and $2 \pi$ giving a total of 8 filters. By combining the energies of the Gabor filters for all the directions and for each resolution a total of 8 features are obtained. In this work, the size of the bone intensity image is $32 \times 32$ and Gabor representation with two scales and eight orientations is considered.

Each image region was decomposed into a wavelet basis of resolution two using a coiflet of order4. This wavelet filter is applied to each of the 65 cropped images, using two different levels of resolution. At each resolution level three detail coefficient matrices were calculated resulting in three matrices representing the vertical, horizontal and diagonal structures of the image. The novel feature sets proposed in this chapter are calculated by applying the magnitude operator to rectify the wavelet coefficients, and then calculating the first and second order moments of the resulting distribution. The wavelet subbands were then preprocessed, by taking the absolute value of each coefficient and binning each detail into sixty four bins. Once the preprocessing was completed, the histogram of each of the detail coefficient matrix was calculated.

A simple and powerful feature extracted from the wavelet co- efficients is the average energy of each detail image. This is defined as the sum of the squares of each detail image, normalized for the total number of coefficients in the image. Such energy signatures provide a good indication of the total energy contained at specific spatial frequency levels and orientation.

The variance of the wavelet coefficients, often known as the energy is defined as

$$
E_{j 1}=\frac{1}{M N} \sum_{m=1}^{M} \sum_{n=1}^{N} D_{j 1}(m, n)^{2}
$$

where $D_{j l}$ is the detail coefficient image 1 at resolution level $j$ of size $(\mathrm{M}, \mathrm{N})$.

a) Wavelet Statistical Features

First, a histogram was calculated from each wavelet detail. The histogram calculated on wavelet coefficients measures the frequency distribution of contrast levels. Mean, Standard Deviation and six first order statistical texture descriptors were then extracted from the histogram of each coefficient matrix. This yields twenty four texture descriptors (eight for each detail) for every level of resolution.

b) Wavelet Co-occurrence Features

Secondly, co-occurrence matrices were calculated at each 
detail and level of resolution. A co-occurrence matrix captures the spatial dependence of contrast values, depending on different directions and distances specified. Four co-occurrence matrices were calculated for each detail matrix at each resolution level. A co-occurrence matrix was calculated for four directions, 0, 45, 90, and 135 degrees at a set distance of one.

Traditional co-occurrence techniques also consider several distances between pixels. Since the texture descriptors are calculated based on multi-resolution wavelets, the resolution levels act as distances. The following nine Haralick texture descriptors were then extracted from each co-occurrence matrix: energy, entropy, contrast, homogeneity, sum-mean, variance, maximum probability, inverse difference moment, and cluster tendency. The final texture descriptor vector had 132 elements per resolution level, generating a 396 element texture descriptor vector per image. Feature reduction is necessary to reduce the feature space so it is manageable for the decision trees. The feature space was limited by decreasing the number of texture descriptors. The size of the texture description vector was reduced to 99 by averaging over the four co-occurrence directions.

c) Wavelet Scale Co-occurrence Features

In each of the feature extraction techniques listed above the coefficients of each subimage are analyzed separately, with the correlations between the subimages of same and different resolution levels ignored, whereas scale co-occurrence matrices capture information about the relationships between each subband of the transform and the low frequency approximation at the corresponding level.In this work relationship between direction and scale subimages of the wavelet transform is considered to quantitatively measure the correlation between each orientation subimage at a given resolution level $\mathrm{j}$, as well as between each detail image and the detail images at neighboring resolution levels. These parameters are then used, along with autocorrelation features of both the magnitude and raw coefficient values and various wavelet based first and second order statistics to classify the bone images.

The features that are considered in the context of scale co-occurrence matrix are inertia, contrast, local homogeneity and correlation. The first two features have the most discriminating power of all the co-occurrence features and capture many aspects of the shape of a co-occurrence matrix. 2) Ridgelets

As the digital ridgelet transform implementation requires prime size image each image slice is therefore further cropped, and only square $31 \times 31$ sub-images fully contained in the implant recipient site are generated. By decomposing the images using DRT, 16 sub-bands are extracted from $31 \times 31$ images. A feature vector consists of energy values extracted at different sub-bands which lead to a feature database for further processing. Several different combinations of resolution levels were investigated, and two levels of resolution were determined to be best.

\section{3) Curvelets}

For this paper we chose the discrete version of the curvelet transform known as 'wrapping algorithm' proposed by Candes and Donoho( ). This approach uses a spatial grid to translate curvelets at each scale and angle, assuming a regular rectangular grid defining 'Cartesian' curvelets. This method applies a 2D fast Fourier transform to the image. For each scale and angle, a product of $\mathrm{U}_{\mathrm{j}}$ is obtained. The result is then wrapped around the origin. The 2D inverse fast Fourier transform is then applied, resulting in discrete curvelet coefficients. Several features were calculated from the curvelet coefficients. Features such as simple energy, mean and standard deviation, curvelet first order statistical features and curvelet co-occurrence features are calculated from each of these curvelet subbands and are stored in the data base for further processing. Each of these statistics was calculated for two levels of resolution and for each curvelet matrix based on each radial 'wedge'(16 angles).

\section{4) Contourlets}

A novel feature extraction algorithm is proposed based on the contourlet decomposition of the image region. The complete algorithm for the proposed framework is as follow

Algorithm :( Extraction of texture features)

1) Decompose each ROI image in the Contourlet domain.

2) At each level '9-7' filters are used for LP decomposition and 'PKVA' filters are used for directional subband decomposition.

3) The energy of the image block associated with each subband is calculated (Refer Equation 4.7 for energy calculation).

4) Compute the standard deviation (SD) of the decomposed image on each directional subband. Standard deviation is given as

$$
\sigma_{\mathrm{k}}=\sqrt{\frac{1}{\mathrm{MxN}} \sum_{\mathrm{i}=1}^{\mathrm{M}} \sum_{\mathrm{j}=1}^{\mathrm{M}}\left(\mathrm{W}_{\mathrm{k}}(\mathrm{i}, \mathrm{j})-\mu_{\mathrm{k}}\right)^{2}}
$$

where $\mathrm{W}_{\mathrm{k}}=$ Coefficients of $\mathrm{k}^{\text {th }} \mathrm{CT}$ decomposed Sub-band $\mu_{\mathrm{k}}=$ Mean value of $\mathrm{k}^{\text {th }}$ Sub-band $\mathrm{MxN}=$ Size of the CT decomposed Sub-band

The resulting SD vector is

$$
\mathrm{f}=\left[\sigma_{1}, \sigma_{2}, \sigma_{3} \ldots \sigma_{\mathrm{n}}\right]
$$

5) Contourlet co-occurrence parameters from each directional subband are computed.

To extract texture features first the image is decomposed into a low pass subband and several band-pass directional subbands. From the decomposition it is clear that only contourlets that match with both location and direction of image contours produce significant coefficients. The feature extraction depends on the choice of filters and number of directions. Hence different combinations of pyramidal (P) filters: the Haar and 9-7 filters, and directional (D) filters: the CD filters (A. Cohen et al 1995) and the ladder filters by Phoong et al (1995) (referred to as PKVA filters) are analyzed.Edge and texture orientations are captured by using contourlet decomposition with a 2 level LP decomposition using 9-7 filter for pyramidal decomposition and PKVA for directional decomposition.

The novel feature sets proposed in this chapter are 
calculated by applying the magnitude operator to rectify the contourlet coefficients, and then calculating the first and second order moments of the resulting distribution. The contourlet directional subbands were then preprocessed, by taking the absolute value of each coefficient and binning each directional sub-band into sixty four bins.

Since directional sub-bands are pure high pass filters, the mean of the contourlet detail coefficients equals zero. Consequently, the energy is exactly their variance. Employing energy as a texture feature is thus equivalent to characterizing the detail sub-band by a Gaussian. Standard deviation vector and energy of each sub-band is used as image feature which is computed on each directional sub-band of the contourlet decomposed image. Results indicate that using a feature vector containing contourlet directional energy features significantly improved discriminatory power compared to the wavelet, ridgelet and curvelet based classification algorithms.

\section{Texture classification}

The classification step was carried out using a decision tree classifier based on C\&RT approach, Support Vector Machine, Adaboost and Bayes classifier.The Bayes classifier is optimal for any problem, as it minimizes the probability of error. The Bayes classifier has found empirical success in

classifying high dimensional data sets.An SVM uses a device called kernel mapping to map the data in input space to a high dimensional feature space in which the problem becomes linearly separable.An important advantage that separates SVM from other classifiers is its principle of structural risk minimization, rather than focusing on empirical risk minimization as used by many other methods.It focuses on minimizing the generalization error, instead of simply minimizing a bound on training error. SVM does not depend explicitly on the dimensionality of the problem, only to the margin with which it separates the data, with all these factors SVM achieves superior performance even on high dimensional data sets.

A decision tree predicts the class of an object (bone type) from values of predictor variables (statistical texture descriptors). The most relevant texture descriptors are found and based on those selected descriptors, a set of decision rules were generated. Using the C\&RT approach, each tree's parameters, were optimized, including depth of tree, number of parent nodes, and number of child nodes. The parameters were considered optimal when the highest possible rate of accuracy was found.

It is a competition-based tree node classification, which categorizes the features into two classes. The other reason for using the tree classification is to reduce the sensitivity of texture analysis to the delineation of abnormal regions when an unknown feature vector is submitted for classification. It will first go to the root node, which is always a decision node and then take one of the two branches based on the outcome of testing one of its features against the threshold at that decision node. This process continues until the feature vector reaches one of the terminal nodes where it is assigned a class.

This tree structured classification approach has several advantages. Firstly, tree classification does automatic stepwise feature selection and complexity reduction; secondly, tree classification is robust with respect to outliers and misclassified points in the training set; thirdly, the final classifier can be compactly stored; fourthly, classification tree efficiently classifies new data; and finally, classification tree provides easily understood and interpreted information regarding the predictive structure of the data.

AdaBoost learning can efficiently solve three fundamental problems (Freund 1996, 1997): (1) Selecting effective features from redundant feature space, (2) Constructing weak classifiers using selected features and (3) Building a strong classifier. Here, we apply it to select the most effective features.

The classification performance of the classifier is then estimated by constructing a confusion matrix. A confusion matrix is a table that lists bone groups and its true positives, true negatives, false positives and false negatives (Table 3.3). The number of true positives is the number of samples that are correctly classified. The number of false positives is the number of samples that are incorrectly classified. The number of true negatives is the number of other samples that are incorrectly classified. The number of false negatives is the number of other samples that are correctly classified. From the misclassification matrix specificity, sensitivity, precision and accuracy statistics were computed.

TABLE I: MEASURES OF CLASSIFICATION PERFORMANCE

\begin{tabular}{|c|c|}
\hline Measure & Definition \\
\hline Sensitivity & True Positives / Total Positives \\
Specificity & True Negatives / Total Negatives \\
Precision & True Positives / Total Positives and \\
Accuracy & FP \\
& TP + TN/Total samples \\
\hline
\end{tabular}

\section{RESULTS}

The discriminating powers of the feature vectors are described in the following sections. Section 4.1 compares the best descriptors for Gabor and Coiflet wavelets. Section 4.2 compares four different feature vectors calculated on the ridgelet and curvelet transforms. Section 4.3 compares four different feature vectors calculated on the contourlet transform. Section 4.4 contains a global comparison of the three best multi-resolution techniques ,as well as comparing them with run-length,co-occurrence and first order statistics.

\section{A. Wavelet comparison}

In comparing the two wavelet-based features Coiflet performed slightly with higher accuracy rates in the 80-95\% compared to Gabor wavelet. Wavelet scale co-occurrence features significantly outperform any of the other features under the classification rule of C\&RT Classifier and SVM for bone texture classification. As was expected, energy and wavelet statistical features did not perform well on the bone texture images, from which it can be concluded that pixel relationships at small distances are insufficient to characterize the texture of a bone image.

It shows that wavelet scale and co-occurrence features perform better than energy features. From this Table 2 it is obvious that the overall best result is not obtained by a single group of parameters but with a carefully chosen combination 
of several groups. Adding multi-resolution information at different scales indeed significantly increases the classifier performance. The combination of the statistical and multi-scale representation of the bone image gives adequate information to classify the different bone groups compared to gray level features at single scale.

TABLE II: CLASSIFICATION PERFORMANCE OF WAVELET AND GABOR TEXTURE FEATURE SET

\begin{tabular}{|c|c|c|c|c|c|}
\hline \multirow{2}{*}{ Feature Set } & \multicolumn{5}{|c|}{ Classification accuracy (\%) } \\
\cline { 2 - 6 } & C\&RT & Adaboost & SVM & $\begin{array}{c}\text { Bayes } \\
\text { Classif } \\
\text { ier }\end{array}$ & LVQ \\
\hline Gabor Energy & 94 & 64 & 88.1 & 88.3 & 53.7 \\
\hline $\begin{array}{c}\text { Wavelet } \\
\text { Energy }\end{array}$ & 80 & 79.5 & 87.1 & 62.9 & 52.8 \\
\hline $\begin{array}{c}\text { Wavelet } \\
\text { Statistical }\end{array}$ & 89.5 & 75 & 83.3 & 65.63 & 55.23 \\
\hline $\begin{array}{c}\text { Wavelet } \\
\text { Co-occurrence }\end{array}$ & 93.75 & 77.08 & 86.06 & 64.58 & 56.16 \\
\hline $\begin{array}{c}\text { Wavelet Scale } \\
\text { co-occurrence }\end{array}$ & 95 & 80.3 & 89 & 80.75 & 67.9 \\
\hline
\end{tabular}

\section{B. Ridgelet \& Curvelet comparison}

Table 3 shows a comparison of the performance of the ridgelet and curvelet based feature vectors based on two levels of resolution. The curvelet energy features were in the range 53-94\% and clearly outperformed all other feature vectors. An analysis of the discriminating power of the curvelet statistical and co-occurrence feature vectors, based on the various resolution levels, was also carried out. The results clearly indicated that individual resolution levels did not have sufficient discriminating power, thus multiple resolutions were needed.Curvelet statistical features accuracy rates were significantly lower at 51-85\%.

TABLE III: Classification Performance of CURVElet Features

\begin{tabular}{|c|c|c|c|c|c|}
\hline \multirow{2}{*}{$\begin{array}{c}\text { Feature } \\
\text { Set }\end{array}$} & \multicolumn{5}{|c|}{ Classification Accuracy(\%) } \\
\cline { 2 - 6 } C\&RT & $\begin{array}{c}\text { Adabo } \\
\text { ost }\end{array}$ & SVM & $\begin{array}{c}\text { Bayes } \\
\text { Classifi } \\
\text { er }\end{array}$ & LVQ \\
\hline $\begin{array}{c}\text { Curvelet } \\
\text { energy }\end{array}$ & 85.8 & 85.3 & 94 & 84.7 & 53.16 \\
\hline $\begin{array}{c}\text { Curvelet } \\
\text { statistical }\end{array}$ & 81.3 & 82 & 85 & 76.21 & 51 \\
\hline $\begin{array}{c}\text { Curvelet } \\
\text { co-occurr } \\
\text { ence }\end{array}$ & 80 & 81.3 & 88 & 81 & 50.83 \\
\hline $\begin{array}{c}\text { Ridgelet } \\
\text { features }\end{array}$ & 81.7 & 72.7 & 82 & 78.6 & 50 \\
\hline
\end{tabular}

\section{Contourlet comparison}

Table 4 shows a comparison of the performance for three contourlet based feature vectors, which clearly illustrates the improved performance of the proposed directional energy feature sets providing the highest classification accuracy rates for a wide variety of classifiers. Features selected by the contourlet based standard deviation perform better, under the classification rule of C\&RT Classifier and Adaboost. From the results it can be seen that co-occurrence features provides with lowest accuracy, which indicates the sign of the coefficients is of importance when calculating second order statistics. The results show that pixel relationships at small distances are insufficient to characterize the texture of a bone image.

TABLE IV: Classification PERFormance of CONTOURLET FEATURES

\begin{tabular}{|l|c|c|c|c|c|}
\hline \multirow{2}{*}{ Feature Set } & \multicolumn{5}{|c|}{ Classification Accuracy (\%) } \\
\cline { 2 - 6 } & C\&RT & Adaboost & SVM & $\begin{array}{c}\text { Bayes } \\
\text { Classifier }\end{array}$ & LVQ \\
\hline $\begin{array}{l}\text { Contourlet } \\
\text { energy }\end{array}$ & 90.63 & 88 & 90 & 87.5 & $\begin{array}{c}56.1 \\
6\end{array}$ \\
\hline $\begin{array}{l}\text { Contourlet } \\
\text { standard } \\
\text { deviation }\end{array}$ & 93.75 & 85.7 & 87.5 & 86.21 & 51.6 \\
\hline $\begin{array}{l}\text { Contourlet } \\
\text { co-occurrence }\end{array}$ & 91.95 & 84 & 84.3 & 81.25 & $\begin{array}{c}54.8 \\
3\end{array}$ \\
\hline $\begin{array}{l}\text { Contourlet } \\
\text { pooled } \\
\text { features }\end{array}$ & 97.69 & 97.6 & 94 & 88.12 & $\begin{array}{c}53.4 \\
5\end{array}$ \\
\hline
\end{tabular}

These results indicate that a combination of energy, standard deviation and co-occurrence features are the most effective descriptors for contourlets.Contourlet pooled features outperformed all other feature vectors, having accuracy rates between $88-98 \%$, which was higher than energy features(in the $88-91 \%$ range), standard deviation features(in the $86-94 \%$ range) and co-occurrence features(in the $81-92 \%$ range).Considering the increased performance using the contourlet descriptors, the use of additional descriptors would be worth exploring.

\section{Global comparison}

Table 5 illustrates a comparison of accuracy results for bone quality for the best wavelet-based (Coiflet), best curvelet-based (energy) and best contourlet-based (pooled) feature sets. The contourlet based descriptors clearly outperforms all wavelet, ridgelet and curvelet-based descriptors.

TABLE : ANALYSIS OF EXISTING ALGORITHMS WITH PROPOSED ALGORITHM

\begin{tabular}{|c|c|c|c|c|c|}
\hline \multirow{2}{*}{ Feature Set } & \multicolumn{5}{|c|}{ Classification accuracy (\%) } \\
\cline { 2 - 6 } & C\&RT & Adaboost & SVM & $\begin{array}{c}\text { Bayes } \\
\text { Classifier }\end{array}$ & LVQ \\
\hline $\begin{array}{c}\text { Contourlet } \\
\text { energy features }\end{array}$ & 90.63 & 88 & 90 & 87.5 & $\begin{array}{c}56.1 \\
6\end{array}$ \\
\hline $\begin{array}{c}\text { Contourlet } \\
\text { pooled features }\end{array}$ & 97.69 & 97.6 & 94 & 88.12 & $\begin{array}{c}53.4 \\
5\end{array}$ \\
\hline $\begin{array}{c}\text { Gabor Energy } \\
\text { Wavelet Scale } \\
\text { co-occurrence }\end{array}$ & 94 & 64 & 88.1 & 88.3 & 53.7 \\
\hline $\begin{array}{c}\text { Curvelet } \\
\text { features }\end{array}$ & 90.77 & 89.9 & 94 & 85.13 & 53.4 \\
\hline Ridgelet features & 81.7 & 72.7 & 82 & 78.6 & 50 \\
\hline
\end{tabular}

The improved performance of the proposed contourlet directional energy feature sets providing the highest classification accuracy rates for a wide variety of classifiers. 
Features selected by the contourlet based standard deviation perform better, under the classification rule of C\&RT Classifier and Adaboost. From the results it can be seen that co-occurrence features provides with lowest accuracy, which indicates the sign of the coefficients is of importance when calculating second order statistics. The results show that pixel relationships at small distances are insufficient to characterize the texture of a bone image.

The bone textures exhibit more diverse directional components ( curves or irregular shapes) which are well retrieved by the contourlets. The directional energy therefore shows how "abruptly" or "smoothly" the image changes at that point, and therefore how likely it is that part of the image represents an edge, as well as how that edge is likely to be oriented. All the edges have been detected in the multidirectional subbands.From a medical point of view the non-invasive texture features generated from CT images enable and facilitate a more accurate preoperative evaluation of implant recipient site which is very much useful for the surgeon in the treatment of patients. The combination of the statistical and multiscale representation of the bone image gives adequate information to classify the different bone groups compared to gray level features at single scale. Thus it can be concluded that the contourlet based multiresolution texture analysis improves the performance of the radiographic methods.

\section{DISCUSSION AND CONCLUSION}

Trabecular micro architecture and structural variations of trabecular bone are important characteristics (anisotropy) that may have significant clinical implications in bone quality assessment. The ability to assess these characteristics non-invasively would be of great importance to implant dentistry.Two well-known mutiresolution methods and the effect of quantization strategies on the overall classification performance of wavelet texture features are investigated. By applying nonlinearity to the wavelet coefficients two feature sets are extracted from first and second order statistics of these transformed coefficients at various resolution levels.

The relationship between neighboring bands of the wavelet transform contains information which is crucial to the adequate characterization of bone texture. By modeling the relationship using scale co-occurrence matrices, a powerful texture descriptor is developed and experimentally shown to outperform features which are extracted independently from each band. Furthermore, by combining these features in optimal manner further improvements in overall classification accuracy are obtained.

The rotation invariance and extraction of directional information from the images are performed by non-separable wavelets that can capture the intrinsic geometrical structures such as smooth contours in medical images by introducing a new factor direction. With the direction taken into account, these transforms are capable of representing the discontinuities of images i.e. edges, contours more efficiently with a fewer number of coefficients. By utilizing directional features the classification results are further improved and the training requirements for each class reduced.
The results demonstrated that a great amount of bone formation, bone loss as well as remodeling occurred within the first year after tooth extraction. It can be concluded that placement of implants should be performed as soon as possible following tooth extraction in order to maintain the dimensions of the alveolar bone.

The texture analysis was able to directly assess trabecular bone structure in individual orientations. The texture energy for each orientation yielded information regarding trabecular bone orientation and density. The texture based approach gives information about bone orientation at a range of scales, whereas histomorphometric parameters do not measure direct information regarding trabecular structure ,specifically the shape and orientation of trabeculae.Texture analysis not only detected deficits in the amount of bone following a loss of teeth but the orientation in which the deficit occurred.

\section{ACKNOWLEDGEMENT}

The authors wish to express appreciation to the staff of Bharat Scans \& Department of Prosthodontics, Rajarajeswari dental college, Bangalore for their help in clinical data collection.

\section{REFERENCES}

[1] Andre Gahleitner, G.Watzek, H.Imhof, "Dental CT: Imaging Technique, anatomy, and pathologic conditions of the jaws", Euro Radio (2003) 23:366-376.

[2] Anderson L, Kurol M. "CT scan prior to installation of Osseo integrated implants in the maxilla". Int J oral maxillofacial Surg 1987; 16:50-55.

[3] A.K.Jain, "Fundamentals of Digital image processing", prentice-hall International Edition, N.J., USA, 1989.

[4] Arivazhagan. S and Ganesan. L., "Texture classification using wavelet transform, Pattern recognition Letters", 2003, pp. 1513-1521.

[5] Candes E.J, Donoho D.L," New tight frames of curvelets and optimal representation of objects with $\mathrm{C}^{2}$ singularities", comm. pure appl.math. Vol.57, No.2, pp-219-266, 2004.

[6] Candes E.J, Demanet C., Donoho D.L., et al "Fast discrete curvelet transforms", Technical report, Applied and computational Mathematics, California institute of technology, 2005

[7] Cawood J I, Howell RA "classification of the edentulous jaws". Int J oral Maxillofacial Surg 17:232-236. (1988)

[8] Delbalro AM, Greiner FG, Cicata M. "Role of diagnostic imaging in evaluation of the dental implant patient". Radio graphics 1194; 14:699-719.

[9] Do M.N., \& Vetterli M, 2003.The Finite Ridgelet Transform for Image Representation. IEEE Transactions on Image Processing, 12, 16 - 28.

[10] Donoho D.L., M.R.Duncan, 2000.Digital Curvelet Transforms strategy, implementation and experiments. In proc aero sense 2000, wavelet application VII,SPIE, 4056, 12-29.

[11] Ellis GL,Wiscovitch JG. Basal cell Aden carcinoma of the major salivary glands. Oral surg oral med oral pathol. 1990; 69(4):461-9.

[12] Haralick R.M., Shanmugam K., Dinstein I., "Texture features for image classification", IEEE Transaction on system man cybernet, vol.8. no.6, 1973, pp 610-621.

[13] Hirshberg A, Buchner A, Mata static tumors to the oral region, an overview. Eur J cancer B oral oncol 1995; 31B (6);355-60.

[14] Kornel.P, Bela, M., Rainer, S., Zalan, D., Zsolt T and Janos F. (1998) Application of Neural Network in medicine, Diag. Med.Tech. 4 (3), 538-546.

[15] Kurabayashi .T, M.Ida et al "Computed tomography in the diagnosis of maxillofacial mass lesions in younger children", Dentomaxillofacial radiology (1998)27,334-340.

[16] Kara, B. \& Watsuji, N, Using Wavelets for Texture Classification.IJCI Proceedings of International Conference on Signal Processing, ISN 1304-2386,2003.

[17] Liang-Kuang Chen, Cheng-Tau Su, Yuh-Feng Tsai "spiral CT: use in evaluating Dental implantation” Chin J Radio 2001:209-214. 
[18] Lekholm O, Zarb G A, "Patient selection and preparation in Brane mark PI, Zarb G A Albrektsson T (Eds) Osseo integration in clinical dentistry, Quintessence, Chicago, PP 199-209 (1985).

[19] Lucia Dattori, Lindsay Semlar." A comparison of wavelet, ridgelet and curvelet-based texture classification algorithms in computed tomography", Computer in Biology and Medicine 37, 2007, 486-498).

[20] Lenglinger FX et al "DentalCT: examination method, radiation dosage and anatomy", Radiology39:1027-1034.

[21] Svarty K, Anderson JE," CT-scanning in the preoperative planning of Osseo integration implants in the maxilla". Int-J oral maxillo fac Surg 1988;17:33-35

[22] Tuceyran M and Jain A.K, Texture analysis, in hand book of pattern recognition and computer vision $\left(2^{\text {nd }}\right.$ edition), world scientific publishing co. Chapter 2.1, 1998.pp 207-248.

[23] Zhen Ma, João Manuel R. S. Tavares, Renato Natal Jorge, T. Mascarenhas, A Review of Algorithms for Medical Image Segmentation and their Applications to the Female Pelvic Cavity, Computer Methods in Biomechanics and Biomedical Engineering, DOI: 10.1080/10255840903131878, Volume 13, Issue 2, pp. 235-246, April 2010.

[24] 24. Zhen Ma, João Manuel R. S. Tavares, R. M. Natal Jorge, A review on the current segmentation algorithms for medical images, 1st International Conference on Imaging Theory and Applications
(IMAGAPP), ISBN: 978-989-8111-68-5, INSTICC Press, pp. 135-140, 5-8 February 2009, Lisboa, Portugal

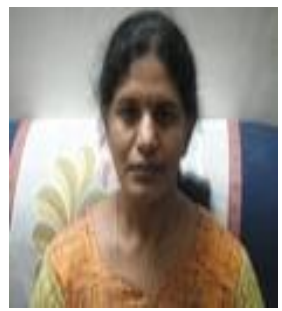

Dr.T. Kalpalatha Reddy is working as a Professor and Dean in Department of Electronics and Communication Engineering, SKR Engineering College (affiliated to Anna University), Chennai, India. She has published several papers in national and international journals and conferences. Her areas of interest are neural networks, medical imaging and bio signal processing. She is a life member of ISTE and Fellow in IETE.

T. Kaliraja is working as a Asst.Professor and Head in Department of Electronics and Communication Engineering, SKR Engineering College (affiliated to Anna University), Chennai, India. He is a life member of ISTE and $\operatorname{IE}(\mathrm{I})$.

B.Ramesh and G.Gurumurthy are working as Lecturers in Department of Electronics and Communication Engineering, SKR Engineering College (affiliated to Anna University), Chennai, India. They are life members of ISTE. 